\title{
BOUNDARY DISTANCE, LENS MAPS AND ENTROPY OF GEODESIC FLOWS OF FINSLER METRICS
}

\author{
DMITRI BURAGO AND SERGEI IVANOV
}

\begin{abstract}
We show that a small perturbation of the boundary distance function of a simple Finsler metric on the $n$-disc is also the boundary distance function of some Finsler metric. (Simple metric form an open class containing all flat metrics.) The lens map is map that sends the exit vector to the entry vector as a geodesic crosses the disc. We show that a small perturbation of a lens map of a simple Finsler metric is in its turn the lens map of some Finsler metric. We use this result to construct a smooth perturbation of the metric on the standard 4dimensional sphere to produce positive metric entropy of the geodesic flow. Furthermore, this flow exhibits local generation of metric entropy, that is positive entropy is generated in arbitrarily small tubes around one trajectory.
\end{abstract}

\section{INTRODUCTION}

In this paper we prove three theorems. At first glance, one of them may seem unrelated to the others, however it heavily depends on them. Rigorous formulations can be found below. Here we give a very informal description to provide the reader with intuition.

We deal with the following situation. We have a disc $D=D^{n}$. This disc is equipped with a Finsler metric. For a geometer, Finsler metrics generalize Riemannian ones. As in the Riemannian case they allow to measure lengths of tangent vectors. However the assumption that this length comes from a quadratic form on the tangent space at every point is dropped. For people from dynamical systems or classical mechanics Finsler metrics are quadratically homogeneous Lagrangians. Thus a Finsler metric determines two structures: a distance function on $D \times D$ and a Lagrangian flow on $T D$.

We work with a class of "nice" Finsler metrics called simple. The precise definition can be found below. To get basic intuition, the reader may think of small smooth perturbations of a flat metric on a Euclidean ball.

Given a simple Finsler metric, we have a number of related objects:

1991 Mathematics Subject Classification. 53C60, 37A35, 37J40.

Key words and phrases. Finsler metric, boundary distance, lens map, scattering relation, Hamiltonian flow, perturbation, metric entropy.

The first author was partially supported by NSF grant DMS-1205997. The second author was supported by Russian Foundation for Basic Research grant 14-01-00062. 
First of all, we have distances between boundary points of the disc. This is a function on $\partial D \times \partial D$ called the boundary distance function associated to the metric inside.

The first question that arises is: if we perturb the boundary distance function, can we find a metric inside which realizes it?

For Riemannian metrics, the answer is no. There is a rather obscure obstruction relying on the Besicovitch inequality [1. Indeed, consider the standard Euclidean unit square. Slightly decrease boundary distances between points close to the opposite vertices. The distances between the pairs of opposite sides remains 1 , therefore in the Riemannian case the area must be at least 1 by the Besicovitch inequality. On the other hand, the main result of [4] implies a slight decrease of the boundary distance function of a flat metric results in a decrease of area. This shows that this boundary distance function cannot be realized by a Riemannian metric.

So far, the authors do not know if a small perturbation of the boundary distance function of a Riemannian metric in a neighborhood of a single pair of points in $\partial D \times \partial D$ can be realized by a Riemannian metric. Here we ignore all distances between pairs of points outside this neighborhood. This seems to be a very intriguing problem.

We are particularly interested in this problem since our dynamical application requires a perturbation localized near one geodesic. We do not know how to make it Riemannian, nor how to do that in low dimension.

We can however prove that a sufficiently small perturbation of the boundary distance function of a simple Finsler metric is the boundary distance function of some Finsler metric. Moreover this Finsler metric is a small perturbation of the original one. See Theorem 1 for the formulation.

The theorem contains two parts. First we work with Finsler metrics without assuming reversibility. The second part, which requires additional effort, provides a construction respecting reversibility. Reversibility of a Finsler metrics means that its Finsler norms are symmetric. Geometers often prefer to assume this symmetry for it gives rise to a usual metric space with a symmetric distance function. In dynamics, there are many situations when one wants to look at non-reversible Finsler metrics. They arise in physics (e.g. magnetic fields) and correspond to non-reversible Lagrangian flows. Non-reversible Finsler metrics determine distance functions that enjoy all axioms of distance other than the symmetry one.

Next we proceed to the lens map (sometimes also called Poincare map or scattering relation). At this point we suggest the reader to think about our disc equipped with a thin collar and some smooth extension of the metric to that collar.

Our Finsler metric determines a Lagrangian flow whose Lagrangian is given by the square of the Finsler norm. Thus we can look at trajectories. They enter our disc. There are entry and exit points for each trajectory of 
the flow. Of course, the flow is defined on the (unit) tangent bundle, hence the entry and exit points are tangent vectors.

We would rather turn to the Hamiltonian language, in which case we think about the entry and exit co-vectors, identified with tangent vectors by the Legendre transform. Thus we get a scattering relation which sends the entry co-vector to the exit co-vector. It is referred to as the dual lens map. This map is symplectic.

There is a very clear relationship between the dual lens map and the boundary distance function of a simple metric. As discussed below, they uniquely determine each other. Namely the derivative of the boundary distance function with respect to its first and second arguments at $p, q \in \partial D$ is essentially the same thing as the entry and exit co-vectors corresponding to the geodesic segment $[p q]$. This follows from the formula for the derivative of the distance function, see (2.1) and (2.2).

Our second result Theorem 2 shows that if one perturbs the dual lens map of a simple Finsler metric in the class of symplectic maps, we also get the dual lens map of a simple Finsler metric.

We hope that these results are of some interest on their own. We also use them to give partial answers to some classic problems in dynamical systems and entropy theory. Here the word "entropy" by default means the measure-theoretic or "metric" entropy.

In our final result Theorem 3 we show that the Riemannian metric of the standard 4-dimensional sphere can be perturbed (in $C^{\infty}$ ) in such a way that the geodesic flow on the unit tangent bundle is of positive entropy. This perturbation is in the class of Finsler metrics, or, equivalently, in the class of Lagrangian systems.

The perturbation takes place in a small tube around one trajectory of the non-perturbed periodic flow. This allows us to give an example in which positive metric entropy is generated in arbitrarily small neighborhoods of one periodic trajectory, see Corollary 5.2 . There is no fixed terminology however many authors call this property entropy non-expansiveness. This property has been of interest of a number of mathematicians including Bowen, Knieper, Newhouse, the first author, see e.g. [2], [3], [6]. In the literature this notion was mostly discussed for topological entropy. Obviously local generation of metric entropy implies that for topological entropy.

Now we proceed with precise definitions and formulations.

Boundary distance. Recall that a Finsler metric on a smooth manifold $M$ is a function $\varphi: T M \rightarrow \mathbb{R}_{+}$which is smooth outside the zero section and such that its restriction on each fiber $T_{x} M, x \in M$, is a Minkowski norm. The latter means that the function $\left.\varphi\right|_{T_{x} M}$ is positively homogeneous of degree 1 , positive outside zero, and its square is quadratically convex.

The word "norm" here is used in a slightly more general sense than in functional analysis, namely we do not assume that it is symmetric. Finsler 
metrics whose norms are symmetric (i.e., $\varphi(-v)=\varphi(v)$ for all $v \in M$ ) are said to be reversible. Whenever we assume reversibily, we mention this explicitly.

The value $\varphi(v)$ is interpreted as the length of a tangent vector $v \in T M$. Then one defines the length of a smooth path and a distance function $d_{\varphi}: M \times M \rightarrow \mathbb{R}$. Similarly to norms, we consider non-symmetric distance functions. By definition, the distance $d_{\varphi}(x, y)$ between points $x, y \in M$ is the infimum of $\varphi$-lengths paths starting at $x$ and ending at $y$. Note that $d_{\varphi}$ satisfies the triangle inequality:

$$
d_{\varphi}(x, y)+d_{\varphi}(y, z) \geq d_{\varphi}(x, z) .
$$

The distance function $d_{\varphi}$ is symmetric if and only of $\varphi$ is reversible.

The boundary distance function $b d_{\varphi}$ of $\varphi$ is the restriction of $d_{\varphi}$ to $\partial M \times$ $\partial M$. That is, $b d_{\varphi}(x, y)$ is the length of a $\varphi$-shortest path in $M$ connecting the boundary points $x$ and $y$.

Geodesics in a Finsler manifold are smooth curves which are locally shortest paths. ¿From dynamical viewpoint, geodesics are trajectories of a Lagrangian system whose Lagrangian is $\frac{1}{2} \varphi^{2}$. We only consider geodesics parametrized by arc length.

Let $D=D^{n}$ denote the $n$-dimensional disc. A Finsler metric $\varphi$ on $D$ is called simple if the following conditions hold:

(1) Every pair of points in $D$ is connected by a unique geodesic.

(2) Geodesics depend smoothly on their endpoints.

(3) The boundary is strictly convex, that is, geodesics never touch it at their interior points.

These conditions imply that all geodesics are minimizing and have no conjugate points.

Our main result Theorem 1 asserts that every $C^{\infty}$-small perturbation of the boundary distance function $b d_{\varphi}$ can be realized as the boundary distance function of a $C^{\infty}$-small perturbation of $\varphi$.

Note that the distance function is not smooth at the diagonal of $D \times D$. To avoid silly technicalities, we work in the following set-up. We only consider perturbations of $b d_{\varphi}$ which are identical in a neighborhood of the diagonal $\Delta$ of $\partial D \times \partial D$, and perturbations of $\varphi$ identical near the boundary.

Theorem 1. Let $\varphi$ be a simple Finsler metric on $D=D^{n}, f=b d_{\varphi}$, and $U_{0}$ a neighborhood of the diagonal $\Delta \subset \partial D \times \partial D$. Then for every function $\widetilde{f}: \partial D \times \partial D \rightarrow \mathbb{R}$ such that $\left.\widetilde{f}\right|_{U_{0}}=\left.f\right|_{U_{0}}$ and $\tilde{f}$ is sufficiently $C^{\infty}$-close to $f$ on $\partial D \times \partial D \backslash U_{0}$ there exists a simple Finsler metric $\widetilde{\varphi}$ such that $b d_{\widetilde{\varphi}}=\widetilde{f}$ and $\widetilde{\varphi}$ coincides with $\varphi$ in a neighborhood of $\partial D$.

The choice of $\widetilde{\varphi}$ can be made in such a way that $\widetilde{\varphi}$ converges to $\varphi$ whenever $\tilde{f}$ converges to $f\left(\right.$ in $\left.C^{\infty}\right)$.

In addition, if $\varphi$ is reversible and $\widetilde{f}$ is symmetric, then $\widetilde{\varphi}$ can be chosen reversible. 
Lens map. Let $\varphi$ be a Finsler metric on $D$. We denote by UTD the unit sphere bundle of $\varphi$. By $U_{i n}$ we denote the set of unit vectors tangent to $D$ at the boundary $\partial D$ and such that they point inwards. Analogously $U_{\text {out }}$ is the set of unit tangent vectors at the boundary pointing outwards.

For a vector $v \in U_{i n}$, we can look at the geodesic with the initial vector $v$. As it hits the boundary again, we get its velocity vector $\beta(v) \in U_{\text {out }}$. This defines a map $\beta: U_{\text {in }} \rightarrow U_{\text {out }}$. This map is the lens map of $\varphi$ (sometimes also called Poincare map or scattering relation). If $\varphi$ is reversible, then the lens map $\beta$ is reversible in the following sense: $-\beta(-\beta(v))=v$ for every $v \in U_{\text {in }}$.

Now we proceed to our next Theorem 2, It answers the question: which perturbations of $\beta$ are realized as lens maps of Finsler metrics? The answer is that the perturbed map should be symplectic. To give a reasonably precise formulation we however need more definitions and notation.

First, to employ the symplectic structure, we need, as usual, to pass to the cotangent bundle $T^{*} D$. Let $\varphi^{*}: T^{*} D \rightarrow \mathbb{R}$ be the fiber-wise dual norm to $\varphi$. That is, for $x \in D$ and $\alpha \in T_{x}^{*} D$, one defines

$$
\varphi^{*}(\alpha)=\sup \left\{\alpha(v) \mid v \in U T_{x} M\right\} .
$$

We denote by $U T^{*} D$ the unit sphere bundle of $\varphi^{*}$.

Let $\mathcal{L}: T D \rightarrow T^{*} D$ be the Legendre transform of the Lagrangian $\frac{1}{2} \varphi^{2}$. Due to homogeneity of $\varphi, \mathcal{L}$ is norm-preserving. In particular it induces a diffeomorphism between $U T D$ and $U T^{*} D$. For a tangent vector $v \in U T_{x} D$, its Legendre transform $\mathcal{L}(v)$ is the unique co-vector $\alpha \in U T_{x}^{*} D$ such that $\alpha(v)=1$.

Then we introduce the dual lens map. Namely consider subsets $U_{i n}^{*}=$ $\mathcal{L}\left(U_{\text {in }}\right)$ and $U_{\text {out }}^{*}=\mathcal{L}\left(U_{\text {out }}\right)$ of $U T^{*} D$. The dual lens map of $\varphi$ is the map $\sigma: U_{\text {in }}^{*} \rightarrow U_{\text {out }}^{*}$ defined by $\sigma=\mathcal{L} \circ \beta \circ \mathcal{L}^{-1}$ where $\beta$ is the lens map of $\varphi$.

Note that $U_{\text {in }}^{*}$ and $U_{\text {out }}^{*}$ are $(2 n-2)$-dimensional submanifolds of $T^{*} D$. Consider the restriction of the canonical symplectic 2-form of $T^{*} D$ to $U_{i n}^{*}$ and $U_{\text {out }}^{*}$. This restriction is non-degenerate and hence symplectic. Indeed, recall that the geodesic flow of $\varphi$ is Hamiltonian when considered on $T^{*} D$. The non-degeneracy claimed above follows from the fact that $U_{\text {in }}^{*}$ and $U_{\text {out }}^{*}$ lie in the energy level of this Hamiltonian flow and transverse to this flow. For the same reason the dual lens map $\sigma$ is symplectic.

If $\varphi$ is reversible then its dual lens map is symmetric in the following sense: $-\sigma(-\sigma(\alpha))=\alpha$ for all $\alpha \in U_{i n}^{*}$. Note that reversibility of $\varphi$ implies that $-\alpha \in U_{\text {out }}^{*}$ if $\alpha \in U_{\text {in }}^{*}$ and vice versa.

Theorem 2. Assume that $n \geq 3$. Let $\varphi$ be a simple metric on $D=D^{n}$ and $\sigma$ its dual lens map. Let $W$ be the complement of a compact set in $U_{i n}^{*}$.

Then every sufficiently small symplectic perturbation $\tilde{\sigma}$ of $\sigma$ such that $\left.\widetilde{\sigma}\right|_{W}=\left.\sigma\right|_{W}$ is realized by the dual lens map of a simple metric $\widetilde{\varphi}$ which coincides with $\varphi$ in some neighborhood of $\partial D$.

The choice of $\widetilde{\varphi}$ can be made in such a way that $\widetilde{\varphi}$ converges to $\varphi$ whenever $\widetilde{\sigma}$ converges to $\sigma\left(\right.$ in $\left.C^{\infty}\right)$. 
In addition, if $\varphi$ is a reversible Finsler metric and $\widetilde{\sigma}$ is symmetric in the sense that $-\widetilde{\sigma}(-\widetilde{\sigma}(\alpha))=\alpha$ for all $\alpha \in U_{i n}^{*}$, then $\widetilde{\varphi}$ can be chosen reversible as well.

Remark. The domain $U_{i n}^{*}$ and range $U_{\text {out }}^{*}$ of the dual lens map depend on the metric $\varphi$. This is not an issue in Theorem 2 because $\widetilde{\varphi}$ is required to coincide with $\varphi$ in a neighborhood of $\partial D$, and the sets $U_{\text {in }}^{*}$ and $U_{\text {out }}^{*}$ are determined by the restriction of $\varphi$ to $\left.T D\right|_{\partial D}$.

Remark. The assumption that $n \geq 3$ in Theorem 2 is essential. In dimension 2 there is a single obstruction, namely the map $\widetilde{\sigma}$ should satisfy certain integral identity, see Remark 4.4 .

Positive entropy. The previous two results are applied to prove the following:

Theorem 3. The standard metric of $S^{4}$ can be perturbed in the class of reversible Finsler metrics so that the resulting metric has positive metric entropy of its geodesic flow. The perturbation can be chosen to be arbitrarily small in $C^{\infty}$.

Note that the analogue of this result for topological entropy is well-known, see [7] and [5].

Acknowledgement. We are grateful to Leonid Polterovich for interesting discussions. In particular he suggested an alternative plan for the final part of the proof of Theorem 3. His approach is purely symplectic. (We do not use it in this paper.) We are grateful to Federico Rodriguez Hertz, Gerhard Knieper and Sheldon Newhouse for inspiring discussions and providing us with references.

\section{Preliminaries}

The following preliminaries are copied with minor modifications from [4].

Let $M=\left(M^{n}, \varphi\right)$ be a Finsler manifold, possibly with boundary. In this paper we only consider the case when $M$ is either the disc $D=D^{n}$ with a simple metric $\varphi$ or an open subset of such a disc.

We say that a continuous function $f: M \rightarrow \mathbb{R}$ is distance-like (with respect to $\varphi)$ if $f$ is smooth on $M \backslash \partial M$ and $\varphi^{*}\left(d_{x} f\right)=1$ for all $x \in M$. Note that every distance-like function $f$ is forward 1-Lipschitz:

$$
f(y)-f(x) \leq d_{\varphi}(x, y)
$$

for all $x, y \in M$.

If $M=D$ and $\varphi$ is a simple metric, then for every $p \in \partial D$ the functions $d_{\varphi}(p, \cdot)$ and $-d_{\varphi}(\cdot, p)$ are distance-like. Note that $d_{\varphi}(\cdot, p)$ is not distance-like in general (unless the metric is reversible).

The Finslerian gradient of a distance-like function $f: M \rightarrow \mathbb{R}$ at $x \in M$, denoted by $\operatorname{grad}_{\varphi} f(x)$, is the unique tangent vector $v \in U_{x} T M$ such that $d_{x} f(v)=1$. Equivalently, $\operatorname{grad}_{\varphi} f(x)=\mathcal{L}^{-1}\left(d_{x} f\right)$ where $\mathcal{L}$ is the Legendre 
transform determined by $\varphi$. Beware that, unlike in the Riemannian case, $\operatorname{grad}_{\varphi}$ is a non-linear operator.

We introduce the following notation for a simple metric $\varphi$ on $D$. For distinct points $x, y \in D$, the unique arc length geodesic segment starting at $x$ and ending at $y$ is denoted by $[x y]$. By $v_{x y}$ and $w_{x y}$ we denote the velocity vectors of this geodesic segment at $x$ and $y$, resp. Thus $v_{x y} \in U T_{x} D$ and $w_{x y} \in U T_{y} D$.

Observe that the $\operatorname{gradient} \operatorname{grad}_{\varphi} d_{\varphi}(x, \cdot)$ at $y$ equals $w_{x y}$. Indeed, the directional derivative of $d_{\varphi}(x, \cdot)$ along $w_{x y}$ equals 1 . Thus the derivative of the function $d_{\varphi}(x, \cdot)$ at $y$ is given by

$$
d_{y} d_{\varphi}(x, \cdot)=\mathcal{L}\left(w_{x y}\right) .
$$

A similar argument for the distance-like function $-d_{\varphi}(\cdot, y)$ shows that

$$
d_{x} d_{\varphi}(\cdot, y)=-\mathcal{L}\left(v_{x y}\right) .
$$

One can see that these formulas are special cases of the First Variation Formula for Finslerian length.

Definition 2.1 (cf. [4]). Let $M=\left(M^{n}, \varphi\right)$ be a Finsler manifold and $S$ a smooth manifold diffeomorphic to $S^{n-1}$. We say that a continuous function $F: S \times M \rightarrow \mathbb{R}$ is an enveloping function for $\varphi$ if $F$ is smooth outside $S \times \partial M$ and the following two conditions are satisfied.

(i) For every $p \in S$, the function $F_{p}:=F(p, \cdot)$ is distance-like;

(ii) For every $x \in M \backslash \partial M$, the map $p \mapsto d_{x} F_{p}$ is a diffeomorphism from $S$ to $U T_{x}^{*} D$.

Note that an enveloping function uniquely determines the metric. Indeed, at every point $x \in M \backslash \partial M$ the unit co-tangent sphere $U T_{x}^{*} M$ can be recovered from $F$ as the image of $S$ under the map $p \mapsto d_{x} F_{p}$. This unit sphere determines the dual norm $\left.\varphi^{*}\right|_{T_{x}^{*} M}$ and hence the original norm $\left.\varphi\right|_{T_{x} M}$.

We need the notion of enveloping function for the following situation. Let $M=D, S=\partial D$ and assume that $\varphi$ is simple. Then the function $F: S \times D \rightarrow \mathbb{R}$ given by $F(p, x)=d_{\varphi}(p, x)$ is an enveloping function for $\varphi$.

The following lemma characterizes enveloping functions of this type.

Lemma 2.2. Let $F: \partial D \times D \rightarrow \mathbb{R}$ be an enveloping function for $\varphi$ such that the following holds.

(i) $F(p, p)=0$ for every $p \in \partial D$;

(ii) For every distinct $p, q \in \partial D$, the function $F_{p}=F(p, \cdot)$ is smooth at $q$ and $\operatorname{grad}_{\varphi} F_{p}(q)$ points outwards $D$.

Then $F(p, x)=d_{\varphi}(p, x)$ for all $p \in \partial D$ and $x \in D$.

Proof. Let $p \in \partial D$ and $x \in D \backslash \partial D$. Consider the backward gradient curve of $F_{p}$ through $x$, namely let $\gamma:\left(-t_{0}, 0\right] \rightarrow D$ be the maximal backward solution of the ODE $\dot{\gamma}(t)=\operatorname{grad}_{\varphi} F_{p}(\gamma(t))$ with the initial condition $\gamma(0)=x$.

Since $F_{p}$ is distance-like, we have $\varphi(\dot{\gamma}(t))=1$ and $\frac{d}{d t} F_{p}(\gamma(t))=1$ for all $t$. Hence $\gamma$ is parametrized by arc length and $F_{p}(\gamma(t))=F_{p}(x)+t$ for all 
$t \in\left(-t_{0}, 0\right]$. Thus for every $t \leq 0$ we have

$$
F_{p}(x)-F_{p}(\gamma(t))=-t=\operatorname{length}_{\varphi}\left(\left.\gamma\right|_{[-t, 0]}\right) \geq d_{\varphi}(\gamma(t), x)
$$

for all $t \in\left(-t_{0}, 0\right]$. On the hand, we have $F_{p}(x)-F_{p}(\gamma(t)) \leq d_{\varphi}(\gamma(t), x)$ since $F_{p}$ is forward 1-Lipschitz. Therefore

$$
F_{p}(x)-F_{p}(\gamma(t))=d_{\varphi}(\gamma(t), x)
$$

for all $t \in\left(-t_{0}, 0\right]$.

Since $F_{p}$ is bounded, (2.3) implies that $\gamma$ is not trapped in $D$ and therefore eventually hits the boundary. Due to (ii), the only point where it may hit the boundary is $p$. Thus $\gamma\left(-t_{0}\right)=p$ and then (2.3) for $t=-t_{0}$ implies that

$$
F_{p}(x)=F_{p}(x)-F_{p}(p)=d_{\varphi}(p, x) .
$$

The lemma follows.

The key point of the proof of Theorem 1 is that a small perturbation of an enveloping function is again an enveloping function for another Finsler metric. The precise statement that we need is the following lemma (borrowed from [4] with minor modifications).

Lemma 2.3. Let $\varphi$ be a simple metric on $D$ and $F: S \times D \rightarrow \mathbb{R}$ an enveloping function for $\varphi$. Let $U \subset D$ be an open set separated from $\partial D$. Then every function $\widetilde{F}: S \times U \rightarrow \mathbb{R}$ which is sufficiently $C^{\infty}$-close to $\left.F\right|_{S \times U}$ is an enveloping function of some Finsler metric $\widetilde{\varphi}$ on $U$.

Furthermore $\widetilde{\varphi}$ tends to $\left.\varphi\right|_{U}$ in $C^{\infty}$ as $\widetilde{F}$ goes to $F$.

Proof. Note that the derivatives of $F$ are bounded on $S \times U$ since $U$ is contained in a compact subset of $D \backslash \partial D$. For every $x \in U$, the map $p \mapsto d_{x} F_{p}$ from $S$ to $T_{x}^{*} D$ parametrizes $U T_{x}^{*} D$ which is a quadratically convex surface in $T_{x}^{*} D$. If $\widetilde{F}$ is close to $F$ then the map $p \mapsto d_{x} \widetilde{F}_{p}$ is close to $p \mapsto d_{x} F_{p}$ and hence it also parametrizes a quadratically convex surface close to the original one. This surface is the unit sphere of some Minkowski norm on $T_{x}^{*} D$. The dual norm on $T_{x} D$ is the desired metric $\widetilde{\varphi}$ at $x$. The entire construction is continuous with respect to relevant $C^{\infty}$ topologies.

\section{Proof of Theorem 1}

Let $n \geq 2, D=D^{n}$ and $S=\partial D$. Let $\varphi, f$ and $\widetilde{f}$ be as in Theorem 1, Recall that $f$ is the boundary distance function of $\varphi$ and $\widetilde{f}$ a perturbation of $f$ supported outside a neighborhood $U_{0}$ of the diagonal $\Delta \subset S \times S$.

For $r>0$ and $p \in D$, let $B_{r}(p)$ denote the forward $d_{\varphi}$-ball of radius $r$ centered at $p$, namely $B_{r}(p)=\left\{x \in D: d_{\varphi}(p, x)<r\right\}$. By $U_{r}(S)$ we denote the forward $r$-neighborhood of the boundary, that is, $U_{r}(S)=\bigcup_{p \in S} B_{r}(p)$.

Since $\tilde{f}$ coincides with $f=b d_{\varphi}$ in a neighborhood $U_{0}$ of the diagonal, there exists $\varepsilon>0$ be such that $\tilde{f}(x, y)=f(x, y)=d_{\varphi}(x, y)$ for all $x, y \in S$ with $d_{\varphi}(x, y)<5 \varepsilon$. Fix fix this $\varepsilon$ for the rest of this section. 
Define a function $F: S \times D \rightarrow \mathbb{R}$ by $F(p, x)=d_{\varphi}(p, x)$. Then $F$ is an enveloping function for $\varphi$. In order prove Theorem 1, we construct an enveloping function $\widetilde{F}: S \times D \rightarrow \mathbb{R}$ for the desired metric $\widetilde{\varphi}$. We make $\widetilde{F}$ out of $F$ and a function $G$ which takes care of a neighborhood of the boundary (see Lemma 3.2 below).

Fix $p \in S$ and define a function $H_{p}: D \rightarrow \mathbb{R}$ by

$$
H_{p}(x)=\max _{y \in S}\left\{\tilde{f}(p, y)-d_{\varphi}(x, y)\right\} .
$$

Note that, if $\tilde{f}=f$ then $H_{p}(x)=d_{\varphi}(p, x)$ and the maximum in (3.1) is attained at the (unique) point $y \in S$ such that $x \in[p y]$.

Though $H_{p}$ is defined on the whole disc $D$, we are going to restrict it to the set $U_{\delta}(S) \backslash B_{\varepsilon}(p)$ where $\delta$ is a sufficiently small positive constant (depending on $\varphi$ and $\varepsilon$ ).

For every $q \in S \backslash\{p\}$, we define a tangent vector $\widetilde{w}_{p q} \in U T_{q} D$ pointing outwards $D$, as follows. Consider the derivative $d_{q} \tilde{f}(p, \cdot) \in T_{q}^{*} S$. If $\tilde{f}=f$, the $\varphi$-norm of this derivative is strictly less than 1. This follows from (2.1) and the fact that the geodesic $[p q]$ is transverse to the boundary. Hence the $\varphi^{*}$-norm of this derivative is less than 1 whenever $\widetilde{f}$ is sufficiently close to $f$ (in $C^{\infty}$, on $S \times S \backslash U_{0}$ ). Hence there exists a unique vector $w \in U T_{q} D$ pointing outwards $D$ and such that $\left.\mathcal{L}(w)\right|_{T_{q} S}=d_{q} \tilde{f}(p, \cdot)$. We take this vector $w$ for $\widetilde{w}_{p q}$. Note that $\widetilde{w}_{p q}$ depends smoothly on $p, q$ and $\widetilde{f}$.

In the case when $\widetilde{f}=f$ we have $\widetilde{w}_{p q}=w_{p q}$ by (2.1) where $w_{p q}$ it the velocity vector of the geodesic $[p q]$ at $q$ (see the notation introduced in Section 2). Therefore $\widetilde{w}_{p q}$ tends to $w_{p q}$ (in $C^{\infty}$, as a function of $p$ and $q$ ), and $\widetilde{w}_{p q}=w_{p q}$ if $d_{\varphi}(p, q)<5 \varepsilon$.

For every distinct $p, q \in S$ and $t \geq 0$ define $\gamma_{p q}(-t)=\exp _{q}\left(-t \widetilde{w}_{p q}\right)$ where $\exp _{q}$ is the exponential map of the metric $\varphi$ at $q$. That is, $\gamma_{p q}$ is a geodesic which hits the boundary at $q$ with velocity $\widetilde{w}_{p q}$. This geodesic is parametrized so that $q=\gamma_{p q}(0)$. The domain of $\gamma_{p q}$ is an interval of the form $[-T, 0]$ where $T$ depends smoothly on $p, q$ and $\tilde{f}$. Hence $T \geq \min \left\{d_{\varphi}(p, q), \varepsilon\right\}$ provided that $\tilde{f}$ is sufficiently close to $f$.

In the case when $\widetilde{f}=f$ we have $\gamma_{p q}=[p q]$. Since $\widetilde{w}_{p q}$ is a $C^{\infty}$-small perturbation of $w_{p q}$, it follows that the geodesics $\left\{\gamma_{p q}\right\}_{q \in S \backslash\{p\}}$ can intersect only within the ball $B_{\varepsilon}(p)$ and cover the set $D \backslash B_{\varepsilon}(p)$.

Lemma 3.1. Let $p, q \in S$ and $t \geq 0$ be such that $\gamma(-t) \in D \backslash B_{\varepsilon}(p)$. Then

$$
H_{p}\left(\gamma_{p q}(-t)\right)=\tilde{f}(p, q)-t,
$$

provided that $\tilde{f}$ is sufficiently close to $f$.

Proof. Let $x=\gamma_{p q}(-t)$. Then $d_{\varphi}(x, q)=t$. Let $y \in S$ be a point of maximum in (3.1). Since the derivative at a point of maximum is zero, we have

$$
\left.d_{y}\left(\tilde{f}(p, \cdot)-d_{\varphi}(x, \cdot)\right)\right|_{T_{y} S}=0 .
$$


Hence

$$
d_{y} \widetilde{f}(p, \cdot)=\left.d_{y} d_{\varphi}(x, \cdot)\right|_{T_{y} S}=\left.\mathcal{L}\left(w_{x y}\right)\right|_{T_{y} S}
$$

where the second equality follows from (2.1). This implies that $w_{x y}=\widetilde{w}_{p y}$, therefore $x$ belongs to the geodesic $\gamma_{p y}$. This implies that $y=q$ because otherwise the geodesics $\gamma_{p q}$ and $\gamma_{p y}$ do not intersect outside $B_{\varepsilon}(p)$.

Thus $q$ is a point of maximum in (3.1). Now (3.1) takes the form

$$
H_{p}(x)=\widetilde{f}(p, q)-d_{\varphi}(x, q)=\widetilde{f}(p, q)-t
$$

and the lemma follows.

Since for a fixed $p$ the geodesics $\gamma_{p q}$ cover $D \backslash B_{\varepsilon}(p)$, the identity (3.2) uniquely determines the function $H_{p}$ in $D \backslash B_{\varepsilon}(p)$. Moreover, the map $(q, t) \mapsto \gamma_{p q}(-t)$ is a diffeomorphism from an appropriate subset of $S \times \mathbb{R}_{+}$ to $D \backslash B_{\varepsilon}(p)$. Hence (3.2) implies that $H_{p}$ is smooth on $D \backslash B_{\varepsilon}(p)$.

Now let us show that $\left.H_{p}\right|_{D \backslash B_{\varepsilon}(p)}$ is a distance-like function. First observe that $H_{p}$ is forward 1-Lipschitz. Indeed, $H_{p}$ is the supremum of functions of the form $x \mapsto \widetilde{f}(p, y)-d_{\varphi}(x, y)$, each of which is forward 1-Lipschitz by the triangle inequality for $d_{\varphi}$. Next, (3.2) implies that $H_{p}$ grows at unit rate along each geodesic $\gamma_{p q}$ within $D \backslash B_{\varepsilon}(p)$. Hence $\left.H_{p}\right|_{D \backslash B_{\varepsilon}(p)}$ is distance-like and

$$
\operatorname{grad}_{\varphi} H_{p}\left(\gamma_{p q}(-t)\right)=\dot{\gamma}_{p q}(-t)
$$

whenever $\gamma_{p q}(-t) \in D \backslash B_{\varepsilon}(p)$.

Lemma 3.2. There exist a neighborhood $V \subset D$ of $S$ and a function $G: S \times$ $V \rightarrow \mathbb{R}$ such that

1. $G$ is an enveloping function for $\left.\varphi\right|_{V}$.

2. $G$ coincides with $\widetilde{f}$ on $S \times S$.

3. $G$ coincides with $F$ in a neighbourhood of $\Delta$ in $S \times V$. (Recall that $\Delta$ is the diagonal of $S \times S$.)

4. For every two different points $p, q \in S$, the gradient of the function $G_{p}=G(p, \cdot)$ at $q$ points outwards $D$.

Furthermore, $G$ is constructed out of $\varphi$ and $\tilde{f}$ in such a way that $G$ converges to $\left.F\right|_{S \times V}\left(\right.$ in $\left.C^{\infty}\right)$ as $\tilde{f}$ goes to $f$.

Proof. Since the boundary is strictly convex with respect to $\varphi$, there exists $\delta=\delta(\varphi, \varepsilon)>0$ such that the following holds. If $p, q \in S, x \in[p q] \cap U_{\delta}(S)$ and $\varepsilon \leq d_{\varphi}(p, x) \leq 2 \varepsilon$, then $d_{\varphi}(p, q)<3 \varepsilon$. Let $V=U_{\delta}(S)$.

For each $p \in S$ define a function $G_{p}: V \rightarrow \mathbb{R}$ by

$$
G_{p}(x)= \begin{cases}H_{p}(x) & \text { if } d_{\varphi}(p, x)>\varepsilon \\ d_{\varphi}(p, x) & \text { if } d_{\varphi}(p, x)<2 \varepsilon\end{cases}
$$

The domains in the two cases in (3.5) overlap, yet the two formulas yield the same value. Indeed, let $x \in V$ be such that $\varepsilon<d_{\varphi}(p, x)<2 \varepsilon$ and let 
$q \in S$ be such that $x \in[p q]$. Then $d_{\varphi}(p, q)<3 \varepsilon$ by the choice of $\delta$. Then $\widetilde{w}_{p q}=w_{p q}$ and hence $\gamma_{p q}=[p q] \ni x$. By Lemma 3.1 it follows that

$$
H_{p}(x)=\tilde{f}(p, q)-d_{\varphi}(x, q)=d_{\varphi}(p, q)-d_{\varphi}(x, q)=d_{\varphi}(p, x) .
$$

Thus the two formulas in (3.5) agree on the overlap. Hence (3.5) defines a continuous function $G_{p}: V \rightarrow \mathbb{R}$.

Define $G: S \times V \rightarrow \mathbb{R}$ by $G(p, x)=G_{p}(x)$. Clearly $G$ is smooth outside $\Delta$ and $G$ tends to $\left.F\right|_{S \times V}$ as $\widetilde{f}$ goes to $f$. We are going to show that $G$ satisfies the assertions of the lemma. The third assertion is trivial by construction.

To prove the second assertion, consider $p, q \in S$. If $d_{\varphi}(p, q)<2 \varepsilon$ then $G(p, q)=d_{\varphi}(p, q)=\widetilde{f}(p, q)$ by (3.5) and the choice of $\varepsilon$. In the other case, namely if $d_{\varphi}(p, q) \geq 2 \varepsilon$, we have $G(p, q)=H_{p}(q)=\widetilde{f}(p, q)$ by setting $t=0$ in Lemma 3.1 .

The fourth assertion follows from the fact that $\operatorname{grad}_{\varphi} G_{p}(q)=\widetilde{w}_{p q}$ for all distinct $p, q \in S$. In the case $d_{\varphi}(p, q)>\varepsilon$ this fact follows from (3.4), and in the case $d_{\varphi}(p, q) \leq \varepsilon$ we have $\operatorname{grad}_{\varphi} G_{p}=w_{p q}=\widetilde{w}_{p q}$.

It remains to verify the first assertion of the lemma. First observe that $G_{p}$ is distance-like because both $H_{p}$ and $d_{\varphi}(p, \cdot)$ are distance-like on $V$. Let $x \in V \backslash S$. Since $G_{p}$ is distance-like, we have a smooth map $p \mapsto d_{x} G_{p}$ from $S$ to $U T_{x}^{*} D$. It remains to prove that this map is a diffeomorphism. Recall that $G$ is a $C^{\infty}$-small perturbation of $\left.F\right|_{S \times V}$ and the map $p \mapsto d_{x} F_{p}$ is a diffeomorphism from $S$ to $U T_{x}^{*} D$. Furthermore $F$ and $G$ coincide within the set $\Delta_{2 \varepsilon}=\{(p, x):|p x|<2 \varepsilon\}$. Hence $d_{x} G_{p}=d_{x} F_{p}$ for $(p, x) \in \Delta_{2 \varepsilon}$. Outside $\Delta_{2 \varepsilon}$ the derivatives of $F$ are uniformly bounded. Hence a sufficiently small perturbation supported outside $\Delta_{2 \varepsilon}$ yields a diffeomorphism $p \mapsto d_{x} G_{p}$ from $S$ to $U T_{x}^{*} D$.

Proof of Theorem 1. Let $G: S \times V \rightarrow \mathbb{R}$ be a function constructed in Lemma 3.2. We glue the desired function $\widetilde{F}$ out of $F$ and $G$ using a partition of unity. Let $h: D \rightarrow[0,1]$ be a smooth function such that $h=1$ outside $V$ and $h=0$ in a neighborhood $V_{0}$ of $S$. Define $\widetilde{F}: S \times D \rightarrow \mathbb{R}$ by

$$
\widetilde{F}(p, x)=h(x) F(p, x)+(1-h(x)) G(p, x) .
$$

Then $\widetilde{F}$ is an enveloping function for a Finsler metric $\widetilde{\varphi}$ which coincides with $\varphi$ within $V_{0}$. Indeed, $\widetilde{F}=G$ in $V_{0} \subset V$ and hence Lemma 3.2 implies that $\widetilde{F}$ is an enveloping function for $\varphi$ within $V_{0}$. Outside $V_{0}, \widetilde{F}$ converges to $F$ (in $\left.C^{\infty}\right)$ as $\widetilde{f} \rightarrow f$. Hence by Lemma 2.3 it is an enveloping function of some Finsler metric $\widetilde{\varphi}$ which is close to $\varphi$.

Since $\widetilde{F}$ is an extension of $\widetilde{f}$, Lemma 2.2 implies that $\widetilde{f}$ is the boundary distance function of $\widetilde{\varphi}$. This finishes the proof of Theorem 1 in the nonreversible case.

In order to make $\widetilde{\varphi}$ reversible (provided that $\varphi$ is reversible and $\widetilde{f}$ is symmetric) we need some preparations. 
Notation 3.3. For a set $U \subset S \times S$ denote by $\Gamma(U)$ the set of velocity vectors of all geodesics $[p q]$ such that $(p, q) \in U$.

Remark 3.4. The construction of $\widetilde{\varphi}$ also guarantees the following property which we need below. If $U \subset S \times S$ is an open set such that $\left.\widetilde{f}\right|_{U}=\left.f\right|_{U}$, then $\widetilde{\varphi}$ coincides with $\varphi$ on $\Gamma(U)$.

Indeed, let $(p, q) \in U$ and $x \in[p q]$. The argument in Step 2 of the proof of Lemma 3.2 shows that every local maximum in (3.1) is global. Hence $H_{p}(x)=F(p, x)$. Going through the subsequent parts of the construction one sees that $\widetilde{F}(p, x)=F(p, x)$. Since $U$ is open, this argument also shows that $\widetilde{F}\left(p^{\prime}, x\right)=F\left(p^{\prime}, x\right)$ for all $p^{\prime}$ from a neighborhood of $p$ in $S$. Therefore $\widetilde{\varphi}^{*}$ coincides with $\varphi^{*}$ in a neighborhood of the co-vector $d_{x} F_{p}$. Let $v$ be the velocity vector of $[p q]$ at $x$. Then $d_{x} F_{p}$ is the Legendre transform of $v$. Hence $\widetilde{\varphi}(v)=\varphi(v)$ as claimed.

Now we are in position to prove the last assertion of Theorem 1, Let $R$ denote the involution of $S \times S$ permuting its arguments, i.e., $R(p, q)=(q, p)$. Recall that $\tilde{f}$ and $f$ coincide in a neighborhood $U_{0}$ of the diagonal $\Delta \subset S \times S$. Fix an open covering $\left\{U_{i}\right\}_{i=1}^{N}$ of $S \times S \backslash U_{0}$ such that for every $i$ the sets $U_{i}$ and $R\left(U_{i}\right)$ have disjoint closures.

Connect $f$ to $\tilde{f}$ by a sequence of functions $f=f_{0}, f_{1}, \ldots, f_{N}=\tilde{f}$ where every $f_{i}: S \times S \rightarrow \mathbb{R}$ is a symmetric function obtained from $f_{i-1}$ by a $C^{\infty}$ small perturbation supported in $U_{i} \cup R\left(U_{i}\right)$. By induction, we construct a sequence of reversible metrics $\varphi_{i}$ on $D$ such that $f_{i}$ is the boundary distance function of $\varphi_{i}$ for each $i$. The induction step goes as follows. First apply Theorem 11 to $\varphi_{i-1}$ in place of $\varphi$ and $f_{i}$ in place of $\widetilde{f}$, resp. This yields a non-reversible metric $\widetilde{\varphi}_{i}$ whose boundary distance function is $f_{i}$. By Remark 3.4. $\widetilde{\varphi}_{i}$ coincides with $\varphi_{i-i}$ outside $\Gamma\left(U_{i} \cup R\left(U_{i}\right)\right)$ where $\Gamma(\ldots)$ is defined as in Notation 3.3 however with respect to $\varphi_{i-1}$ in place of $\varphi$. Since $\Gamma\left(U_{i}\right)$ and $\Gamma\left(R\left(U_{i}\right)\right)$ have disjoint closures, we can change the metric within $\Gamma\left(R\left(U_{i}\right)\right)$ so that it becomes reversible. Namely we define the desired metric $\varphi_{i}$ by

$$
\varphi_{i}(v)= \begin{cases}\widetilde{\varphi}_{i}(-v) & \text { if } v \in \Gamma\left(R\left(U_{i}\right)\right), \\ \widetilde{\varphi}_{i}(v) & \text { otherwise, }\end{cases}
$$

for every $v \in U T D$. This metric has the same boundary distance function. Indeed, if $(p, q) \notin R\left(U_{i}\right)$ then the geodesic $[p q]$ of the metric $\widetilde{\varphi}_{i}$ remains a geodesic as we replace $\widetilde{\varphi}_{i}$ by $\varphi_{i}$. Hence $d_{\varphi_{i}}(p, q)=d_{\widetilde{\varphi}_{i}}(p, q)=f_{i}(p, q)$ if $(p, q) \notin R\left(U_{i}\right)$. This identity holds for $(p, q) \in R\left(U_{i}\right)$ because in this case $(q, p) \notin R\left(U_{i}\right)$ and the distance is symmetric.

It remains to verify that the set of functions $\tilde{f}$ reachable by sequences $f_{0}, \ldots, f_{N}$ such that the construction works, contains a neighborhood of $f$ in our space of boundary distance functions. (Recall that this is the space functions on $S \times S$ coinciding with $f$ in $U_{0}$, equipped with the $C^{\infty}$ topology.) The covering $\left\{U_{i}\right\}$ and hence the number of steps in the construction it determined by $U_{0}$. The construction which produces $\widetilde{\varphi}$ out of $\varphi$ and $\widetilde{f}$ is 
explicit and hence continuous in its arguments $\varphi$ and $\widetilde{f}$ (regarded as elements of respective functional spaces). Furthermore it is defined for an open set of pairs $(\varphi, \widetilde{f})$. The decomposition and symmetrization procedures above also enjoy similar continuity properties. Therefore if $\tilde{f}$ is chosen from a suitable neighborhood of $b d_{\varphi}$ then each pair $\left(\varphi_{i-1}, f_{i}\right)$ in the inductive construction belongs to the domain where Theorem 1 applies.

\section{Proof of Theorem 2}

As in the previous sections, let $\varphi$ be a simple metric on $D=D^{n}$ and $S=\partial D$. Recall that in Theorem 2 we assume that $n \geq 3$. The metric $\varphi$ (or its restriction to a neighborhood of $S$ ) determines the sets $U_{\text {in }}^{*}, U_{\text {out }}^{*} \subset T^{*} D$ as in Section 1 .

For the proof of the theorem we need a zoo of notations. The good news is that they have clear geometric meaning.

We think of trajectories of a Hamiltonian flow on the unit co-tangent bundle $U T^{*} D$. They enter $U T^{*} D$ and leave it. Thus we have an entry covector in $U_{i n}^{*}$ and an exit co-vector in $U_{\text {out }}^{*}$ for each trajectory. Of course each of them can be viewed as a pair consisting of the base point in $\partial D$ and the co-vector component.

A diffeomorphism $\sigma: U_{\text {in }}^{*} \rightarrow U_{\text {out }}^{*}$ tells us the exit co-vector from the entry one. In the following notations and constructions we use only certain properties of $\sigma$ and do not assume that it arose from a flow. We apply these constructions to both $\sigma$ and $\widetilde{\sigma}$.

First we need a map $P_{\sigma}$ which takes an entry co-vector and produces two points $p, q \in \partial D$ which are the entry and exit base points of the "trajectory" of $\sigma$ determined by that co-vector. Analogously, $Q_{\sigma}$ takes an exit co-vector and produces the entry and exit base points.

Formally, we define maps $P_{\sigma}: U_{\text {in }}^{*} \rightarrow S \times S$ and $Q_{\sigma}: U_{\text {out }}^{*} \rightarrow S \times S$ by

$$
P_{\sigma}(\alpha)=(\pi(\alpha), \pi(\sigma(\alpha))), \quad \alpha \in U_{i n}^{*},
$$

and

$$
Q_{\sigma}(\alpha)=\left(\pi\left(\sigma^{-1}(\alpha)\right), \pi(\alpha)\right), \quad \alpha \in U_{\text {out }}^{*},
$$

where $\pi: T^{*} D \rightarrow D$ is the bundle projection. Note that $P_{\sigma}=Q_{\sigma} \circ \sigma$.

Next we need the inverse maps $P_{\sigma}^{-1}$ and $Q_{\sigma}^{-1}$. The map $P_{\sigma}^{-1}$ takes two distinct points from the boundary $\partial D$ as its input and tells us the entry co-vector. Similarly, $Q_{\sigma}^{-1}$ takes two distinct boundary points and reports the exit co-vector. In order to ensure that $P_{\sigma}^{-1}$ and $Q_{\sigma}^{-1}$ exist we assume that $\sigma$ is nice in the following sense:

Definition 4.1. We say that a map $\sigma: U_{\text {in }}^{*} \rightarrow U_{\text {out }}^{*}$ is nice if the following conditions are satisfied:

(1) $\sigma$ is a symplectic diffeomorphism between $U_{\text {in }}^{*}$ and $U_{\text {out }}^{*}$.

(2) $P_{\sigma}$ is a diffeomorphism between $U_{i n}^{*}$ and $S \times S \backslash \Delta$.

(3) $Q_{\sigma}$ is a diffeomorphism between $U_{\text {out }}^{*}$ and $S \times S \backslash \Delta$.

Here, as in the previous sections, $\Delta$ denotes the diagonal of $S \times S$. 
In fact, the third condition in Definition 4.1 follows from the second one and the identity $P_{\sigma}=Q_{\sigma} \circ \sigma$. We include them both in the definition for the clarity of exposition. The assumption that $\sigma$ is symplectic is crucial. Nonetheless it is used only in one place, namely in the proof of Lemma 4.3.

If $\sigma$ is the dual lens map of a simple metric then $\sigma$ is nice. Furthermore if $\widetilde{\sigma}$ is obtained from a nice map $\sigma$ by a sufficiently small compactly supported perturbation (as in Theorem 2), then $\widetilde{\sigma}$ is nice as well.

The last bit of notation is 1 -form $\lambda_{\sigma}$ on $S \times S \backslash \Delta$ defined as follows. For $p, q \in S, p \neq q, \xi \in T_{p} S, \eta \in T_{q} S$, define

$$
\lambda_{\sigma}(\xi, \eta)=-P_{\sigma}^{-1}(p, q)(\xi)+Q_{\sigma}^{-1}(p, q)(\eta) .
$$

Here $(\xi, \eta)$ is regarded as an element of $T_{(p, q)}(S \times S \backslash \Delta)$ through the standard identification $T(S \times S) \simeq T S \times T S$. The maps $P_{\sigma}^{-1}$ and $Q_{\sigma}^{-1}$ in (4.1) are correctly defined since $\sigma$ is nice. Moreover $P_{\sigma}^{-1}(p, q) \in\left(U_{i n}^{*}\right)_{p}$ and $Q_{\sigma}^{-1}(p, q) \in\left(U_{\text {out }}^{*}\right)_{q}$ where $\left(U_{\text {in }}^{*}\right)_{p}$ and $\left(U_{\text {out }}^{*}\right)_{q}$ are the fibers of $U_{\text {in }}^{*}$ and $U_{\text {out }}^{*}$ over $p$ and $q$, resp. Therefore the terms $P_{\sigma}^{-1}(p, q)(\xi)$ and $Q_{\sigma}^{-1}(p, q)(\eta)$ in (4.1) make sense.

The following lemma explains where the definition of $\lambda_{\sigma}$ comes from. The lemma tells us that if $\sigma$ arises from a simple metric then $\lambda_{\sigma}$ is the derivative of the distance function, as follows:

Lemma 4.2. Let $f=b d_{\varphi}$ and let $\sigma: U_{\text {in }}^{*} \rightarrow U_{\text {out }}^{*}$ be a nice map. Then $\sigma$ is the dual lens map of $\varphi$ if and only if $d f=\lambda_{\sigma}$ on $S \times S \backslash \Delta$.

Proof. We begin with the "only if" part. Let $\sigma$ be the dual lens map of $\varphi$. Let $p, q \in S, p \neq q, \alpha=\mathcal{L}\left(v_{p q}\right)$ and $\beta=\mathcal{L}\left(w_{p q}\right)$ where $v_{p q}$ and $w_{p q}$ are the velocity vectors of the geodesic $[p q]$ at $p$ and $q$, see notation in Section 2 , Then $\beta=\sigma(\alpha)$ and $P_{\sigma}(\alpha)=Q_{\sigma}(\beta)=(p, q)$. By (2.2) and (2.1) we have $d_{p} f(\cdot, q)=-\alpha$ and $d_{q} f(p, \cdot)=\beta$. Hence for every $\xi \in T_{p} S$ and $\eta \in T_{q} S$,

$$
d f(\xi, \eta)=-\alpha(\xi)+\beta(\eta)=-P_{\sigma}^{-1}(p, q)(\xi)+Q_{\sigma}^{-1}(p, q)(\eta)=\lambda_{\sigma}(\xi, \eta) .
$$

This proves the "only if" part of the lemma.

To prove the "if" part, let $\widehat{\sigma}$ be the dual lens map of $\varphi$. By the "only if" part we have $d f=\lambda_{\widehat{\sigma}}$ on $S \times S \backslash \Delta$. Thus it suffices to prove that $\sigma=\widehat{\sigma}$ provided that $\lambda_{\sigma}=\lambda_{\widehat{\sigma}}$.

Let $\alpha \in U_{\text {in }}^{*}, p=\pi(\alpha) \in S, \beta=\widehat{\sigma}(\alpha) \in U_{\text {out }}^{*}$ and $q=\pi(\beta) \in S \backslash\{p\}$. Then $P_{\widehat{\sigma}}(\alpha)=Q_{\widehat{\sigma}}(\beta)=(p, q)$. Since $\lambda_{\widehat{\sigma}}=\lambda_{\sigma}$, substituting $\eta=0$ into (4.1) yields that

$$
P_{\sigma}^{-1}(p, q)(\xi)=P_{\widehat{\sigma}}^{-1}(p, q)(\xi)=\alpha(\xi)
$$

for every $\xi \in T_{p} S$. That is, $\left.P_{\sigma}^{-1}(p, q)\right|_{T_{p} S}=\left.\alpha\right|_{T_{p} S}$. Since $P_{\sigma}^{-1}(p, q) \in\left(U_{i n}^{*}\right)_{p}$ and a co-vector from $\left(U_{i n}^{*}\right)_{p}$ is uniquely determined by its restriction to $T_{p} S$, it follows that $P_{\sigma}^{-1}(p, q)=\alpha$. Hence $\sigma(\alpha) \in\left(U_{\text {out }}^{*}\right)_{q}$.

Similarly, substituting $\xi=0$ into (4.1) yields that $\left.Q_{\sigma}^{-1}(p, q)\right|_{T_{q} S}=\left.\beta\right|_{T_{q} S}$ and therefore $Q_{\sigma}^{-1}(p, q)=\beta$. Hence $\sigma^{-1}(\beta) \in\left(U_{i n}^{*}\right)_{p}$. Thus

$$
\pi\left(\sigma^{-1}(\beta)\right)=p=\pi(\alpha)=\pi\left(\sigma^{-1}(\sigma(\alpha))\right)
$$


Since $\sigma$ is nice, $Q_{\sigma}$ is a diffeomorphism from $U_{\text {out }}^{*}$ to $S \times S \backslash \Delta$. In particular, the restriction $\left.Q_{\sigma}\right|_{\left(U_{\text {out }}^{*}\right)_{q}}$ is a diffeomorphism from $\left(U_{\text {out }}^{*}\right)_{q}$ to $(S \backslash\{q\}) \times\{q\}$. Hence the first coordinate map of this restriction, namely $\left.\pi \circ \sigma^{-1}\right|_{\left(U_{\text {out }}^{*}\right)_{q}}$, is injective. Since $\beta \in\left(U_{\text {out }}^{*}\right)_{q}$ and $\sigma(\alpha) \in\left(U_{\text {out }}^{*}\right)_{q}$, this injectivity and (4.2) imply that $\sigma(\alpha)=\beta$. Since $\alpha$ is an arbitrary element of $U_{i n}^{*}$ and $\beta=\widehat{\sigma}(\alpha)$, it follows that $\sigma=\widehat{\sigma}$. This finishes the proof of the "if" part of the lemma.

Lemma 4.3. $\lambda_{\sigma}$ is a closed 1-form for every nice map $\sigma: U_{\text {in }}^{*} \rightarrow U_{\text {out }}^{*}$.

Proof. Let $\nu$ denote the canonical 1-form on $T^{*} D$. Recall that the canonical 1-form is defined as follows: for $\alpha \in T^{*} D$ and $\tau \in T_{\alpha} T^{*} D$,

$$
\nu(\tau)=\alpha(d \pi(\tau))
$$

where $\pi: T^{*} D \rightarrow D$ is the bundle projection. The two terms in (4.1) are actually pull-backs of $\nu$ by $P_{\sigma}^{-1}$ and $Q_{\sigma}^{-1}$, namely

$$
\lambda_{\sigma}=-\left(P_{\sigma}^{-1}\right)^{*} \nu+\left(Q_{\sigma}^{-1}\right)^{*} \nu \text {. }
$$

Hence

$$
d \lambda_{\sigma}=-\left(P_{\sigma}^{-1}\right)^{*}(d \nu)+\left(Q_{\sigma}^{-1}\right)^{*}(d \nu) .
$$

Let $\omega$ be the canonical symplectic form on $T^{*} D$. Recall that $\omega=d \nu$. Therefore

$$
\begin{aligned}
d \lambda_{\sigma} & =-\left(P_{\sigma}^{-1}\right)^{*} \omega+\left(Q_{\sigma}^{-1}\right)^{*} \omega=-\left(P_{\sigma}^{-1}\right)^{*} \omega+\left(\sigma \circ P_{\sigma}^{-1}\right)^{*} \omega \\
& =-\left(P_{\sigma}^{-1}\right)^{*} \omega+\left(P_{\sigma}^{-1}\right)^{*}\left(\sigma^{*} \omega\right)=0
\end{aligned}
$$

Here we use the identity $P_{\sigma}=Q_{\sigma} \circ \sigma$ and the fact that $\sigma^{*} \omega=\omega$ because $\sigma$ is symplectic. Thus $d \lambda_{\sigma}=0$ and the lemma follows.

Proof of Theorem Q Now let $\sigma$ be the dual lens map of $\varphi$ and $\widetilde{\sigma}$ be a perturbation of $\sigma$ as in Theorem 2. Recall that $\widetilde{\sigma}$ is nice provided that the perturbation is sufficiently small. Then by Lemma 4.3 the 1-form $\lambda_{\widetilde{\sigma}}$ is closed. Since $n \geq 3$, the space $S \times S \backslash \Delta$ is simply connected and therefore $\lambda_{\widetilde{\sigma}}$ is exact. Hence there exists a smooth function $\tilde{f}: S \times S \backslash \Delta \rightarrow \mathbb{R}$ such that $d \widetilde{f}=\lambda_{\widetilde{\sigma}}$.

Since $\widetilde{\sigma}$ coincide with $\sigma$ outside a compact set, $\lambda_{\widetilde{\sigma}}$ and $\lambda_{\sigma}$ also coincide outside a compact subset of $S \times S \backslash \Delta$. That is, there exists a tubular neighborhood $U$ of $\Delta$ in $S \times S$ such that $\left.\lambda_{\widetilde{\sigma}}\right|_{U \backslash \Delta}=\left.\lambda_{\sigma}\right|_{U \backslash \Delta}$. This fact and Lemma 4.2 imply that

$$
\left.d \widetilde{f}\right|_{U \backslash \Delta}=\left.d f\right|_{U \backslash \Delta}
$$

where $f$ is the boundary distance function of $\varphi$. Since $n \geq 3$, the set $U \backslash \Delta$ is connected. This and (4.3) imply that $\tilde{f}-f$ is constant on $U \backslash \Delta$. Since $\tilde{f}$ is defined up to an additive constant, we may assume that $\widetilde{f}=f$ on $U \backslash \Delta$. Now we can extend $\widetilde{f}$ to the whole $S \times S$ by setting it zero on $\Delta$. Then $\widetilde{f}$ is a small perturbation of $f$ in the sense of Theorem 1 Hence by Theorem 1 there exists a simple Finsler metric $\widetilde{\varphi}$ whose boundary distance function is $\widetilde{f}$. 
Moreover $\widetilde{\varphi}$ coincides with $\varphi$ in a neighborhood of the boundary and is close to $\varphi$ in $C^{\infty}$. Now Lemma 4.2 applied to $\widetilde{\varphi}$ in place of $\varphi$ implies that $\widetilde{\sigma}$ is the dual lens map of $\sigma$.

It remains to prove the last assertion of Theorem 2, namely that $\widetilde{\varphi}$ can be chosen reversible if $\varphi$ is reversible and $\widetilde{\sigma}$ satisfies the symmetry condition: $-\tilde{\sigma}(-\widetilde{\sigma}(\alpha))=\alpha$ for all $\alpha \in U_{i n}^{*}$. By the last assertion of Theorem 1, it suffices to verify that $\widetilde{f}$ is symmetric, that is, $\widetilde{f}(p, q)=\widetilde{f}(q, p)$ for all $p, q \in S$. First observe that the symmetry condition on $\widetilde{\sigma}$ implies that

$$
P_{\widetilde{\sigma}}^{-1}(q, p)=-Q_{\widetilde{\sigma}}^{-1}(p, q)
$$

for all $p, q \in S, p \neq q$. Indeed, if $\alpha=P_{\widetilde{\sigma}}^{-1}(p, q)$ and $\beta=Q_{\widetilde{\sigma}}^{-1}(p, q)$ then $\widetilde{\sigma}(\alpha)=\beta$, hence $\widetilde{\sigma}(-\beta)=-\alpha$ by the symmetry condition. Therefore $P_{\widetilde{\sigma}}^{-1}(q, p)=-\beta$ and (4.4) follows.

Now consider the function $\widetilde{g}: S \times S \rightarrow \mathbb{R}$ defined by $\widetilde{g}(x, y)=\widetilde{f}(y, x)$. Let $p, q \in S, p \neq q, \xi \in T_{p} S$ and $\eta \in T_{q} S$. Then

$$
\begin{aligned}
d_{(p, q)} \widetilde{g}(\xi, \eta)=d_{(q, p)} \tilde{f}(\eta, \xi) & =-P_{\widetilde{\sigma}}^{-1}(q, p)(\eta)+Q_{\widetilde{\sigma}}^{-1}(q, p)(\xi) \\
& =Q_{\widetilde{\sigma}}^{-1}(p, q)(\eta)-P_{\widetilde{\sigma}}^{-1}(p, q)(\xi)=d_{(p, q)} \widetilde{f}(\xi, \eta) .
\end{aligned}
$$

Here we used the identity $d \widetilde{f}=\lambda_{\tilde{\sigma}}$, the definition of $\lambda_{\widetilde{\sigma}}$ and (4.4). Thus $d \widetilde{g}=d \widetilde{f}$, hence $\widetilde{g}=\widetilde{f}$, that is, $\widetilde{f}$ is symmetric. This finishes the proof of Theorem 2 ,

Remark 4.4. The above argument does not work in dimension $n=2$ for two reasons. The first issue is that $S \times S \backslash \Delta$ is not simply connected so the closed 1-form $\lambda_{\widetilde{\sigma}}$ a priori may not be exact. However it is easy to see that this 1-form is exact even in dimension 2. Indeed, the fundamental group of $S \times S \backslash \Delta$ is cyclic and its generator can be realized by a loop arbitrarily close to $\Delta$. Since $\lambda_{\widetilde{\sigma}}$ and $\lambda_{\sigma}$ coincide in a neighborhood of $\Delta$, their integrals over this loop are equal. Since $\lambda_{\sigma}=d f$ is exact, these integrals are zero. Hence $\lambda_{\widetilde{\sigma}}$ is exact as well.

The second issue with $n=2$ is that $U \backslash \Delta$ is not connected, where $U$ is a tubular neighborhood of $\Delta$ is $S \times S$. In fact, $U \backslash \Delta$ consists of two components. These components are represented by points $\left(p, q_{1}\right)$ and $\left(p, q_{2}\right)$ in $S \times S \backslash \Delta$ where $p \in S$ is an arbitrary point and $q_{1}, q_{2} \in S$ are close to $p$ and lie on different sides of it. It may happen that the function $\tilde{f}$ (an antiderivative of $\lambda_{\widetilde{\sigma}}$ ) approaches different values as we tend to the diagonal $\Delta$ from different sides. In this case $\widetilde{f}$ cannot be extended to the whole $S \times S$ as a continuous function. To rule out this situation we need to impose an additional condition on $\widetilde{\sigma}$, namely

$$
\int_{\{p\} \times(S \backslash\{p\})} \lambda_{\widetilde{\sigma}}=0
$$

for some (and then all) $p \in S$. This condition is a necessary and sufficient one and it is easy to give examples when it is not satisfied. 
Taking into account the definition of $\lambda_{\widetilde{\sigma}}$, the condition (4.5) can be interpreted as follows. For a fixed $p \in S$, the image $\widetilde{\sigma}\left(\left.U_{i n}^{*}\right|_{p}\right)$ is a graph of a differential 1-form on $S \backslash\{p\}$, and (4.5) is equivalent to the requirement that the (improper) integral of this 1-form over $S \backslash\{p\}$ is zero.

\section{Proof of Theorem 3}

In this section we deduce Theorem 3 from Theorem 2, Consider the unit disc $D^{6} \subset \mathbb{R}^{6}$ equipped with the standard symplectic structure. We need the following lemma.

Lemma 5.1. There exists a symplectomorphism $\theta: D^{6} \rightarrow D^{6}$ which is arbitrarily close to the identity in $C^{\infty}$, coincides with the identity map near the boundary, and has positive metric entropy.

Proof. Let $M$ be a surface of genus $\geq 2$ equipped with a Riemannian metric of negative curvature. Let $I_{1}=(-1,1), I_{2}=(-2,2)$ and $N=M \times I_{2}$. Let $B^{*} M$ denote the bundle of unit balls in $T^{*} M$.

First we construct a compactly supported Hamiltonian $H: T^{*} N \rightarrow \mathbb{R}$ whose Hamiltonian flow has positive metric entropy. Let $\psi: \mathbb{R} \rightarrow \mathbb{R}_{+}$be a smooth cut-off function which equals 1 on $[-1,1]$ and vanishes outside $\left(-\frac{3}{2}, \frac{3}{2}\right)$. For a co-vector $(x, y, \xi, \eta) \in T^{*} N$ where $x \in M, y \in I_{2}, \xi \in T_{x}^{*} M$, $\eta \in T_{y}^{*} I_{2}$, we define

$$
H(x, y, \xi, \eta)=\psi(y) \cdot \psi(|\xi|) \cdot \psi(|\eta|) \cdot \frac{|\xi|^{2}}{2}
$$

where $|\xi|$ is the norm of $\xi$ determined by the Riemannian metric of $M$.

Observe that $H$ is compactly supported. Consider the Hamiltonian flow of $H$ restricted to the set

$$
B^{*} M \times B^{*} I_{1} \subset T^{*} M \times T^{*} I_{2}=T^{*} N .
$$

On this set we have $H(x, y, \xi, \eta)=|\xi|^{2} / 2$. Recall that the standard Hamiltonian of our Riemannian metric is given by $H_{\text {Riem }}(x, \xi)=|\xi|^{2} / 2$. Hence the Hamiltonian flow of $H$ restricted to $B^{*} M \times B^{*} I_{1}$ is the product of the geodesic flow of our Riemannian metric (restricted to $B^{*} M$ ) and the trivial flow on $B^{*} I_{1}$. Hence the metric entropy of this flow is positive.

Now let $U \subset \mathbb{R}^{3}$ be an open set diffeomorphic to $N$ (namely $U$ is a thickened handlebody). A diffeomorphism between $N$ and $U$ induces a symplectomorphism between $T^{*} N$ and $T^{*} U$. This symplectomorphism sends the Hamiltonian flow of $H$ to a compactly supported Hamiltonian flow on $T^{*} U \subset T^{*} \mathbb{R}^{3} \simeq \mathbb{R}^{6}$. The support of this flow fits into $D^{6}$ upon a suitable rescaling.

Thus there exists a Hamiltonian flow $\left\{\Phi^{t}\right\}$ on $D^{6}$ which vanishes near the boundary and has positive metric entropy. Finally, in order to construct the desired symplectomorphism $\theta$, we take a map $\Phi^{t}$ for a sufficiently small $t>0$. 
Proof of Theorem 3. Consider the sphere $S^{4}$ with its standard Riemannian metric and let $D \subset S^{4}$ be a small geodesic ball. Then $D$ is diffeomorphic to $D^{4}$ and the metric of $S^{4}$ restricts to a simple Riemannian metric on $D$. Using the notations introduced before Theorem 2, consider the dual lens map $\sigma: U_{\text {in }}^{*} \rightarrow U_{\text {out }}^{*}$.

Fix a co-vector $\alpha \in U_{i n}^{*}$ and let $\omega=\beta^{*}(\alpha)$. Let $V \Subset U_{i n}^{*}$ be a neighborhood of $\alpha$ in $U_{i n}^{*}$. We choose $V$ to be symplectomorphic to a rescaled copy of the interior of the standard disc $D^{6}$. By Lemma 5.1 there exists a compactly supported symplectomorphism $\theta: V \rightarrow V$ with positive metric entropy and arbitrarily close to the identity map in the $C^{\infty}$ topology. We extend $\theta$ to a map from the entire $U_{i n}^{*}$ to itself so that it is identity on $U_{i n}^{*} \backslash V$. We use the same letter $\theta$ for this extension.

Define a perturbed dual lens map $\widetilde{\sigma}: U_{\text {in }}^{*} \rightarrow U_{\text {out }}^{*}$ by $\widetilde{\sigma}=\sigma \circ \theta$. If $\theta$ is sufficiently close to identity, then by Theorem 2 the map $\widetilde{\sigma}$ can be realized as the dual lens map of a Finsler metric $\widetilde{\varphi}$. Furthermore $\widetilde{\varphi}$ can be chosen close to the original Riemannian metric and coinciding with it in a neighborhood of the boundary of $D$. We paste the metric $\widetilde{\varphi}$ on $D$ with the original metric on $S^{4} \backslash D$ and obtain a smooth Finsler metric on $S^{4}$. This metric is the desired perturbation with positive entropy.

Indeed, since the original geodesic flow is periodic, the return map of the perturbed geodesic flow equals $\theta$ on $V$ (and remains the identity elsewhere). Thus the perturbed flow contains an invariant open set where the flow is a suspension of $\theta$. Hence the perturbed geodesic flow has positive metric entropy.

The disc $D$ in the proof of Theorem 3 can be chosen arbitrarily small. Thus the perturbation of the geodesic flow is confined to an arbitrarily small neighborhood of one (periodic) orbit of the original flow. This allows us to construct an example with local generation of metric entropy:

Corollary 5.2. There exists a smooth perturbation of the standard metric of $S^{4}$ (in the class of reversible Finsler metrics) such that the following holds. There is a periodic trajectory $\gamma$ such that for every $\varepsilon>0$ the $\varepsilon$-neighborhood of $\gamma$ contains an open invariant set in which the metric entropy is positive. In particular, the flow is entropy non-expansive in the sense of [2].

Proof. Begin with a sequence $\left\{\gamma_{i}\right\}$ of disjoint geodesics in $S^{4}$ converging to a geodesic $\gamma$. For each $i$ choose a neighborhood $U_{i}$ of $\gamma_{i}$ in such a way that these neighborhoods are disjoint. Construct a small perturbation of the metric within each $U_{i}$ as in Theorem 3 . These perturbations should be so small that their derivatives go to zero as $i \rightarrow \infty$. Then the union of these perturbations provides the desired example.

Note that both metric and topological entropy in the $\varepsilon$-neighborhoods in Corollary 5.2 must vanish as $\varepsilon \rightarrow 0$. This follows from the fact that $\varepsilon$-local entropy of any $C^{\infty}$ flow tends to zero as $\varepsilon \rightarrow 0$, see [6]. 


\section{REFERENCES}

[1] A. S. Besicovitch, On two problems of Loewner, J. London Math. Soc. 27 (1952), 141-144.

[2] R. Bowen, Entropy-expansive maps, Trans. Amer. Math. Soc. 164 (1972), 323-331.

[3] D. Burago, A new approach to the computation of the entropy of geodesic flow and similar dynamical systems, Soviet Math. Doklady 37 (1988), 1041-1044.

[4] S. Ivanov, Local monotonicity of Riemannian and Finsler volume with respect to boundary distances, Geometriae Dedicata 164 (2013), no. 1, 83-96; arXiv:1109.4091.

[5] G. Knieper, H. Weiss, A surface with positive curvature and positive topological entropy, J. Differential Geom. 39 (1994), no. 2, 229-249.

[6] S. Newhouse, Continuity properties of entropy, Ann. of Math. 129 (1989), no. 2, 215-235.

[7] G. Paternain, Real analytic convex surfaces with positive topological entropy and rigid body dynamics, Manuscripta Math. 78 (1993), no. 4, 397-402.

Dmitri Burago: Pennsylvania State University, Department of MathematICS, University PARK, PA 16802, USA

E-mail address: burago@math.psu.edu

Sergei Ivanov: St.Petersburg Department of Steklov Mathematical Institute, Russian Academy of Sciences, Fontanka 27, St.Petersburg 191023, Russia

E-mail address: svivanov@pdmi.ras.ru 